\title{
Formation and Rearragement of Vortex Tubes in a 3D Mesoscopic Superconductor with a Central Weak Link
}

\author{
YuAn JiaO ${ }^{a}$, Lin Peng ${ }^{a} *$, YANyAn Zhu ${ }^{a}$, Li Xu ${ }^{a}$, Ying $\mathrm{WANG}^{a}$, Yahua Hu ${ }^{b}$ \\ AND YUN ZHOU ${ }^{c}$ \\ ${ }^{a}$ Department of Physics and College of Environmental and Chemical Engineering, \\ Shanghai University of Electric Power, Shanghai, 201300, China \\ ${ }^{b}$ College of Nanhu, Jiaxing University, Jiaxing, Zhejiang 314001, PR China \\ ${ }^{c}$ Department of Physics, China Jiliang University, Hangzhou, 310018, China
}

(Received October 24, 2017; in final form June 28, 2018)

\begin{abstract}
Vortex patterns are determined for a three-dimensional mesoscopic superconductor with one central weak link, using the time-dependent Ginzburg-Landau equations. The vortex states are obtained for field orientation parallel and perpendicular to the weak link, and vortex rearrangements are found to occur in the vortex patterns. Tilting of the field leads to interesting phenomena caused by the small volume-to-surface ratio, as vortex tubes are preferentially oriented along the field direction while they are forced to be perpendicular to the surface. In addition, we can also observe the intersection of vortex tubes inside (or near) the weak link.
\end{abstract}

DOI: 10.12693/APhysPolA.134.493

PACS/topics: three-dimensional mesoscopic superconductor, time-dependent Ginzburg-Landau equations, finite element method, vortex state

\section{Introduction}

Mesoscopic superconductor exhibit very peculiar magnetic properties when their size is comparable to the coherence length $\xi$ [1]. The mesoscopic samples of different shapes surrounded by vacuum or an insulator medium have been considered extensively both experimentally and theoretically [2-10]. In experiment, the measured superconducting phase boundary for the mesoscopic $\mathrm{Al}$ square shows the direct experimental evidence for these symmetry-induced vortex-antivortex pairs [11]. Recently, an experimental investigation was made of flux jumps and irreversible magnetization of mesoscopic $\mathrm{Al}$ superconducting rings, which indicated that the change of vorticity with magnetic field could be larger than unity [12]. A direct observation of vortex states in small superconducting disks for vorticity $L=0$ to 40 was also reported [13]. Theoretically, it has been shown that many different kinds of superconducting states can exist in mesoscopic samples [2, 5-11]. Vortices also show very rich static and dynamic behavior in the presence of a weak link $[7,10,14]$. It is well known properties of both superconducting and normal metals are modified due to the proximity effect [15] when they are in contact with each other. Thus, the vortex-vortex interaction and the vortex interaction with different kinds of defects and interfaces become very complex.

Recently, we investigated the nanosized superconducting strips with one central weak link using the finite element method to solve the time-dependent Ginzburg-

\footnotetext{
*corresponding author; e-mail: plpeng@shiep.edu.cn
}

Landau (TDGL) equations. Our results indicated that the energy barrier for vortices to enter a weak link is smaller than that for vortices to enter superconducting layers [16]. In addition, we studied the static and dynamic properties of the superconducting condensate in a nanosized type-II superconducting strip with a weakly superconducting narrow metallic region in the presence of external magnetic and applied current [10]. We found that superconducting vortices penetrating the weak-superconducting region are more mobile than the ones in the strong superconducting regions. These studies were based on the two-dimensional limit, where the superconducting condensate was assumed not to vary along the direction of the magnetic field. In this paper, we apply the finite-element method (FEM) to investigate the three-dimensional (3D) cubic superconductor with a central weak link based on the 3D TDGL theory, a proven phenomenological approach for studies of vortex matter in superconductors [17]. We introduce the weakly layered structure in 3D mesoscopic samples, with the objective to understand the penetration of magnetic field in such samples, and the formation and rearrangement of vortex states with respect to the superconducting layers and the sample boundaries.

The paper is organized as follows. In Sect. 2, we show the derived TDGL equations and explain the numerical method and procedure we use in the calculations. In Sect. 3, we analyze the results obtained for the 3D mesoscopic superconductor with one central weak link. Our results are finally summarized in Sect. 4 .

\section{Time-dependent Ginzburg-Landau model}

We consider a mesoscopic superconducting cube of size $a=10 \xi$ with a single weak link of size $d=\xi$. Figure 1 


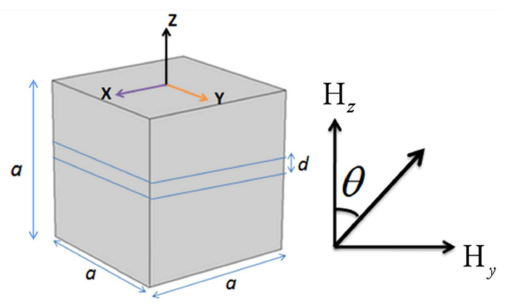

Fig. 1. Coordinate system and the cubic sample.

shows the schematic view of the cylinder under an arbitrarily tilted field, where the rotation angle $\theta$ is measured with respect to the major $(z)$ axis: $H=H_{0} \cos \theta$. The plane of rotation is referred to as the $y-z$ plane, and we introduce the rotation for fields oriented parallel and perpendicular to the major axis: $H_{z} \equiv H\left(\theta=0^{\circ}\right)$ and $H_{y} \equiv H\left(\theta=90^{\circ}\right)$, respectively. The Ginzburg-Landau theory describes the superconducting state through a complex order parameter $\psi$ for which $|\psi|^{2}$ represents the density of the Cooper pairs. In regions where $|\psi|^{2}$ is small, superconductivity is suppressed. At the center of a vortex, $|\psi|^{2}=0$, whereas the local magnetic field $\boldsymbol{B}$ is maximum. The order parameter and the local magnetic field can be determined by their TDGL equations, which are expressed by

$$
\begin{aligned}
& \left(\frac{\partial}{\partial t}+\mathrm{i} \Phi\right) \psi=-(\mathrm{i} \nabla+\boldsymbol{A})^{2} \psi+\left(\zeta-|\psi|^{2}\right) \psi, \\
& \sigma\left(\frac{\partial \boldsymbol{A}}{\partial t}+\nabla \Phi\right)=\boldsymbol{J}_{s}-\kappa^{2} \nabla \times \nabla \times \boldsymbol{A},
\end{aligned}
$$

with boundary conditions

$$
\left.\boldsymbol{n} \cdot(-\mathrm{i} \nabla-\boldsymbol{A}) \psi\right|_{\perp \text { boundary }}=0
$$

where $\Phi$ is the electric potential, $\sigma$ is related to the electric conductivity, $\boldsymbol{n}$ is the normal unit vector on the surface, $\zeta$ is the anisotropy function [10]. The density of the superconducting current $\boldsymbol{J}_{s}$ is given by

$$
\boldsymbol{J}_{s}=\frac{\mathrm{i}}{2}\left(\psi \nabla \psi^{*}-\psi^{*} \nabla \psi\right)-|\psi|^{2} \boldsymbol{A} .
$$

We scale the length in units of $\xi=\frac{\hbar}{\sqrt{2 m\left|\alpha_{0}\right|}}$, the order parameter $\psi$ in units of $\psi_{0}=\sqrt{\frac{-\alpha_{0}}{\beta}}$ (with $\alpha_{0}$ and $\beta$ being the GL coefficients [18]), the vector potential $\boldsymbol{A}$ in units of $A_{0}=\sqrt{2} \kappa H_{c} \xi$, the time $t$ in unit of $t_{0}=\frac{\pi \hbar}{8 k_{\mathrm{B}} T_{c}}$, and the local magnetic field $\boldsymbol{B}=\nabla \times \boldsymbol{A}$ in units of $H_{c 2}=\sqrt{2} \kappa H_{c}$, where $H_{c}$ is the thermodynamic critical field, and $\kappa=\frac{\lambda}{\xi}$ is the GL parameter. Notice that the TDGL equations are gauge invariant under the transformations $\psi^{\prime}=\psi \mathrm{e}^{\mathrm{i} \chi}$, $\boldsymbol{A}^{\prime}=\boldsymbol{A}+\nabla \chi, \Phi^{\prime}=\Phi-\frac{\partial \chi}{\partial t}$. Therefore, we choose the zero-scalar potential gauge, that is, $\Phi=0$ at all times and positions. The free energy of the superconducting state, measured in $F_{0}=\frac{H_{c}^{2} V}{8 \pi}$ units, is expressed as

$$
\begin{aligned}
F & =\frac{2}{V} \int\left[-|\psi|^{2}+\frac{1}{2}|\psi|^{4}+|(\mathrm{i} \nabla+\boldsymbol{A}) \psi|^{2}\right. \\
& \left.+\kappa^{2}(\boldsymbol{B}-\boldsymbol{H})^{2}\right] \mathrm{d} V .
\end{aligned}
$$

We solved these equations starting from zero applied field, which was then increased adiabatically in small steps, $\Delta H=0.01$. The dimensionless magnetization, which is a direct measure of the expelled magnetic field from the sample, is defined as $\boldsymbol{M}=\frac{\langle\boldsymbol{B}\rangle-\boldsymbol{H}}{4 \pi}$, where $\langle\boldsymbol{B}\rangle$ is the magnetic induction averaged over the mesoscopic superconductor volume $V$, i.e., $\langle\boldsymbol{B}\rangle=\frac{1}{V} \int \boldsymbol{B}(\boldsymbol{r}) \mathrm{d} V$. For simplicity, we assume in this work a step-like behavior of $\zeta$ across the system, so that it becomes a coefficient equal to unity inside the superconducting layer, and less than 1 inside the weak link. In our simulations, the weak links are characterized by the anisotropy coefficient $\zeta$ in the GL equation with $\zeta=1$ outside the weak link and $\zeta=0$ in the weak link region. We chose the $\kappa=4$ and $\sigma=1$. The initial conditions are $|\psi|^{2}=1$ corresponding to the Meissner state and zero magnetic field inside the superconductor.

\section{Results and discussions}

We first calculate the full free-energy spectrum, magnetization and the corresponding vortex states as a function of applied magnetic field for the sample with a central weak link. In the first step, the magnetic field is kept parallel to the weak-link plane, and more specifically, parrallel to the $y$ axis $\left(H_{y} \equiv H\left(\theta=90^{\circ}\right)\right)$. Figure 2 a shows the free energy as a function of $H$ for the sample. The lowest energy curve corresponds to the thermodynamic stable
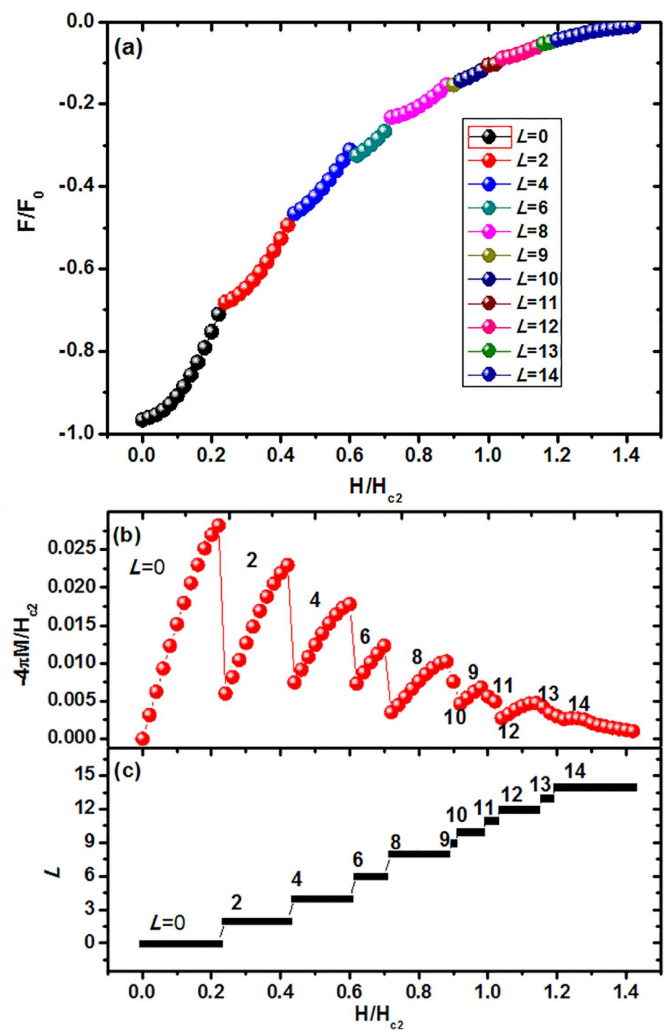

Fig. 2. Free-energy curve (a), magnetization (b) and vorticity (c) for a mesoscopic superconducting cube of size $a=10 \xi$ with a single weak link of size $d=\xi$ in an external uniform magnetic field parallel to the $y$-axis. 
pattern, the Meissner phase with no vortices, but only up to the first penetration field $H_{p}=0.24 H_{c 2}$. Above this field the entrance of two vortex tubes is favored, though it is still possible to keep the system in the Meissner phase but now as a metastable configuration. As the applied field is further raised, four vortex tubes enter the sample.
Each jump in the magnetization curve corresponds to a transition between different vortex states (Fig. 2b, c). We find the multivortex states with vorticity $L=2,4,6,8$, and $9,10, \ldots, 14$ for $0.24 H_{c 2}<H<1.42 H_{c 2}$. We find that there are not $L=1,3,5$, and 7 states existing in the ground states for the cube.
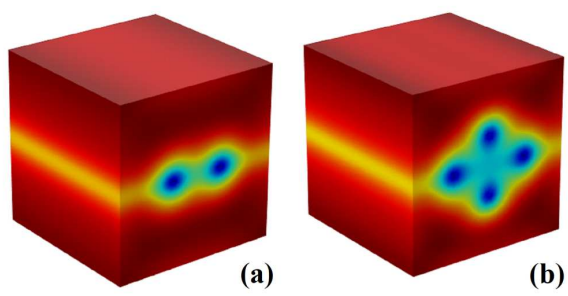

(b)

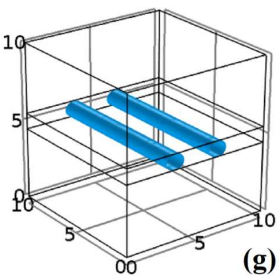

$L=2$
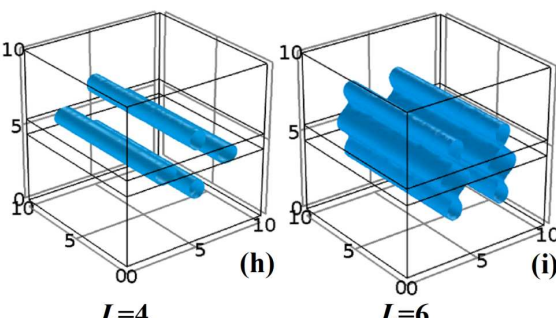

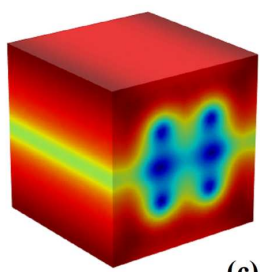

(c)

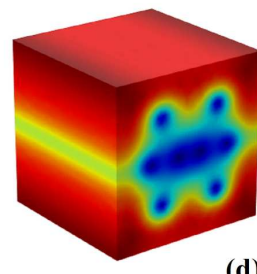

(d)

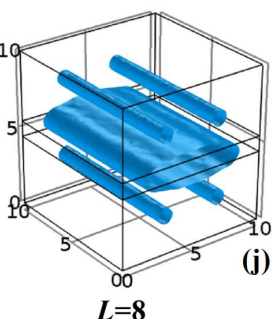

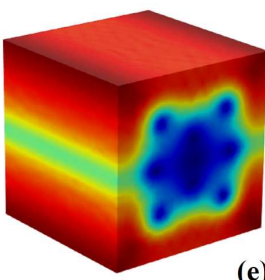

(e)
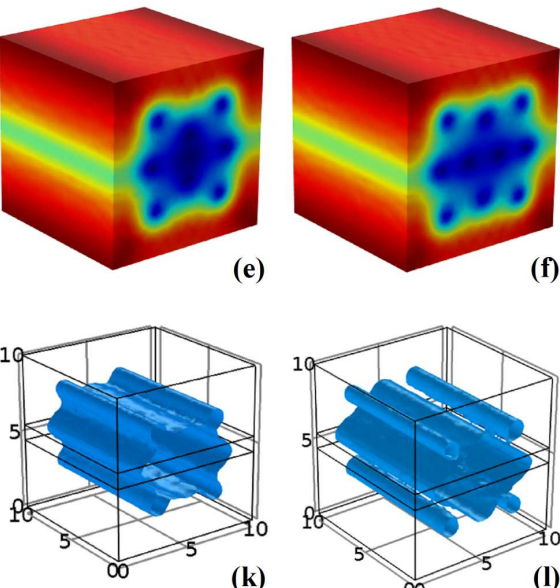

(k)

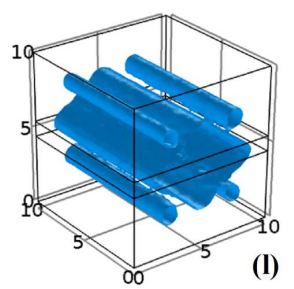

$L=9$

Fig. 3. Vortex tubes for a mesoscopic superconducting cube of size $a=10 \xi$ with a single weak link of size $d=\xi$ in an external uniform magnetic field parallel to the $y$-axis, at the field $\frac{H}{H_{c 2}}=0.34(\mathrm{a}), \frac{H}{H_{c 2}}=0.50(\mathrm{~b}), \frac{H}{H_{c 2}}=0.70(\mathrm{c})$, $\frac{H}{H_{c 2}}=0.78(\mathrm{~d}), \frac{H}{H_{c 2}}=0.90(\mathrm{e})$ and $\frac{H}{H_{c 2}}=0.92(\mathrm{f})$. Parts $(\mathrm{g})-(\mathrm{l})$ show the corresponding isosurface plots of vortex tubes of (a)-(f). Blue to red means order parameter range from minimum to maximum.

Figure 3 shows absolute value of the order parameter and corresponding isosurface plots of vortex tubes for three selected vortex configurations at the field $\frac{H}{H_{c 2}}=$ $0.34, \frac{H}{H_{c 2}}=0.5, \frac{H}{H_{c 2}}=0.7, \frac{H}{H_{c 2}}=0.78$ and $\frac{H}{H_{c 2}}=0.92$. Three selected vortex configurations display vorticity $L=2,4,6,8,9$, and 10 vortex states, respectively. These three vortex configurations illustrate general features of the vortex tubes inside the sample. A vortex tube must reach the surface perpendicularly in order to avoid a supercurrent component pointing outwards the surface. As can be seen in these figures, vortex tubes are located preferably inside the weak link until the saturation number is reached, i.e., these are enough vortex tubes in the weak link so that the increased vortex-vortex interaction expels some of them into the fully superconducting part of the sample. Note also that vortex tubes not only favorably reside in the weak link to minimize energy, but they also enter the sample through the weak link owing to the lower-energy barrier [14].

In what follows, the magnetization $M(H)$ curves are considered in a tilted magnetic field $(H \equiv H(\theta=$ $\left.30^{\circ}\right)$ ) (see Fig. 4). We deliberately separate the magnetic response into the Cartesian components, and would detect a linear combination of $M_{x}, M_{y}$ and $M_{z}$, dependent on its relative position to the sample surfaces. As the applied field increases, we observe the multi-vortex states with vorticity $L=2,4,5,6,7$, and 8 states. Figure 5 shows absolute value of the order parameter and cor-

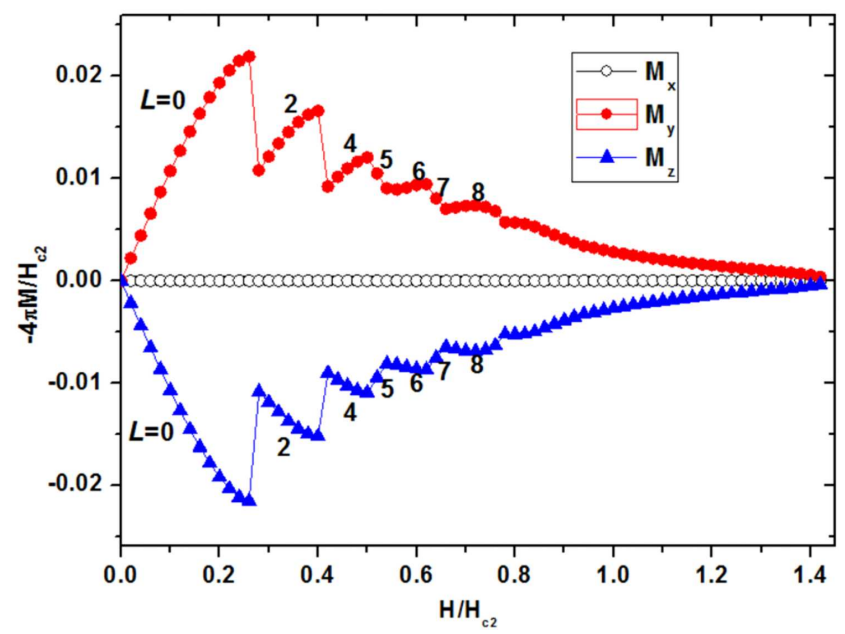

Fig. 4. Magnetization $M(H)$ curves in a tilted magnetic field $\left(H \equiv H\left(\theta=30^{\circ}\right)\right)$. A linear combination of $M_{x}, M_{y}$, and $M_{z}$, dependent on its relative position to the sample surfaces, was detected.

responding isosurface plots of vortex tubes for selected vortex states in a tilted magnetic field for $\theta=30^{\circ}$, at the field $\frac{H}{H_{c 2}}=0.42(\mathrm{a}), \frac{H}{H_{c 2}}=0.52(\mathrm{~b}), \frac{H}{H_{c 2}}=0.58$ (c), $\frac{H}{H_{c 2}}=0.64(\mathrm{~d})$, and $\frac{H}{H_{c 2}}=0.68(\mathrm{e})$. Parts $(\mathrm{f})-(\mathrm{j})$ show the corresponding isosurface plots of vortex tubes of (a)(e). With a magnetic field not parallel to the $x$ or $y$ axis, the vortices attempt to change their orientation ac- 
cordingly. The screening effects of the Meissner currents are always maximal in the corners and vortices, as tubes of magnetic flux must avoid those regions. Therefore, the end of each vortex tube is bent. At the boundaries of the sample, the supercurrent can only have the compo- nent parallel to the surface. Therefore, the vortex endings must be aligned perpendicular to the surface of the sample. On the other hand, we can also observed the intersection of vortex tubes inside (or near) the weak link (see Fig. 5h-j).
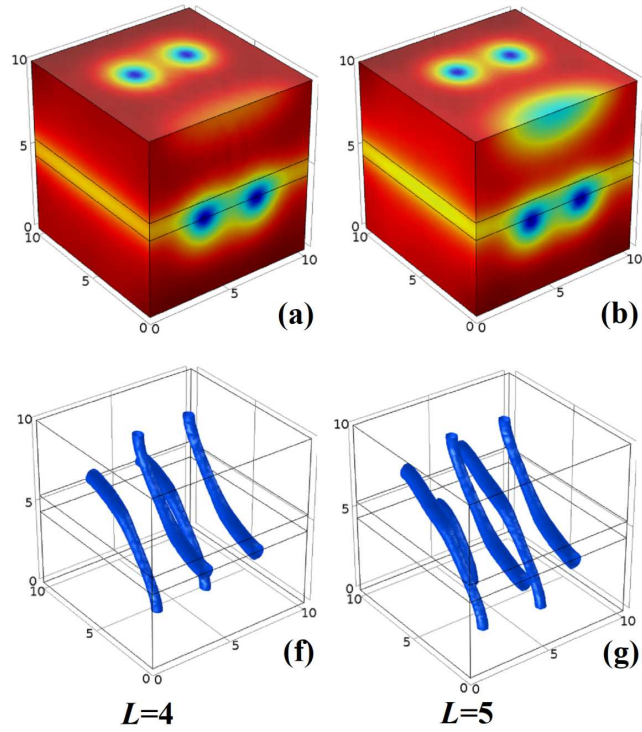

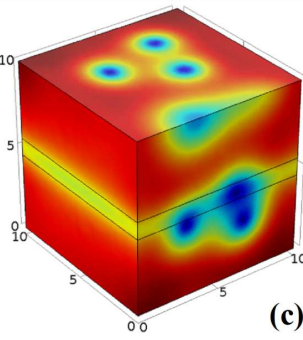

(c)

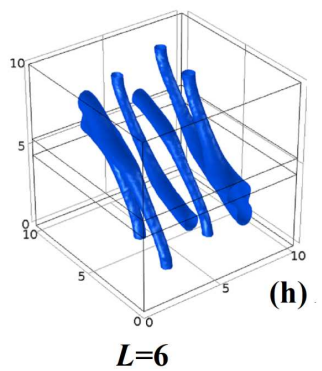

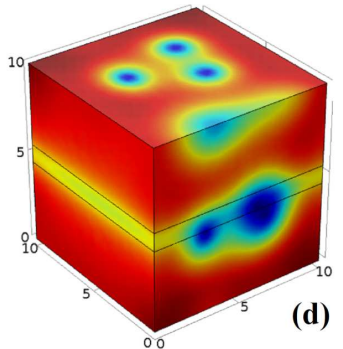

(d)
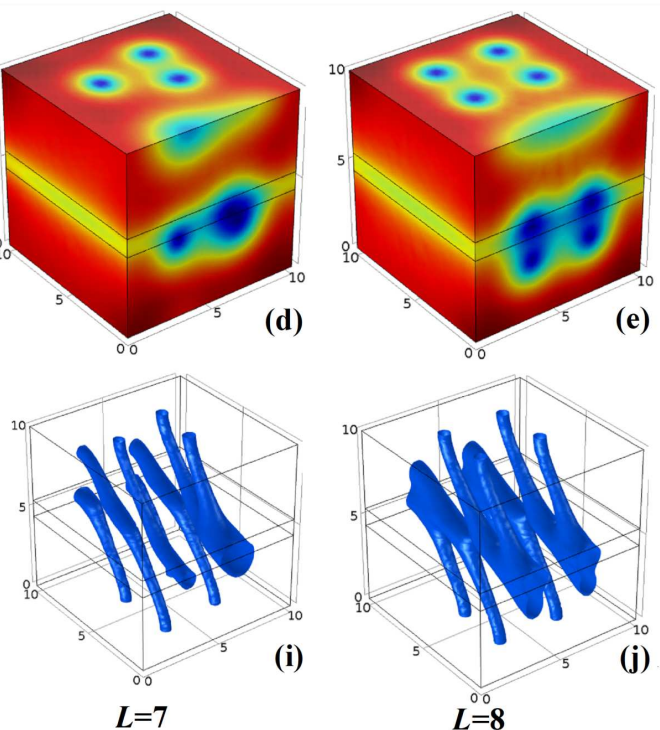

Fig. 5. Absolute value of the order parameter and corresponding isosurface plots of vortex tubes for selected vortex states in a tilted magnetic field for $\theta=30^{\circ}$, at the field $\frac{H}{H_{c 2}}=0.42(\mathrm{a}), \frac{H}{H_{c 2}}=0.52(\mathrm{~b}), \frac{H}{H_{c 2}}=0.58(\mathrm{c}), \frac{H}{H_{c 2}}=0.64(\mathrm{~d})$, and $\frac{H}{H_{c 2}}=0.68$ (e). Parts (f)-(j) show the corresponding isosurface plots of vortex tubes of (a)-(e). Blue to red means order parameter range from minimum to maximum.

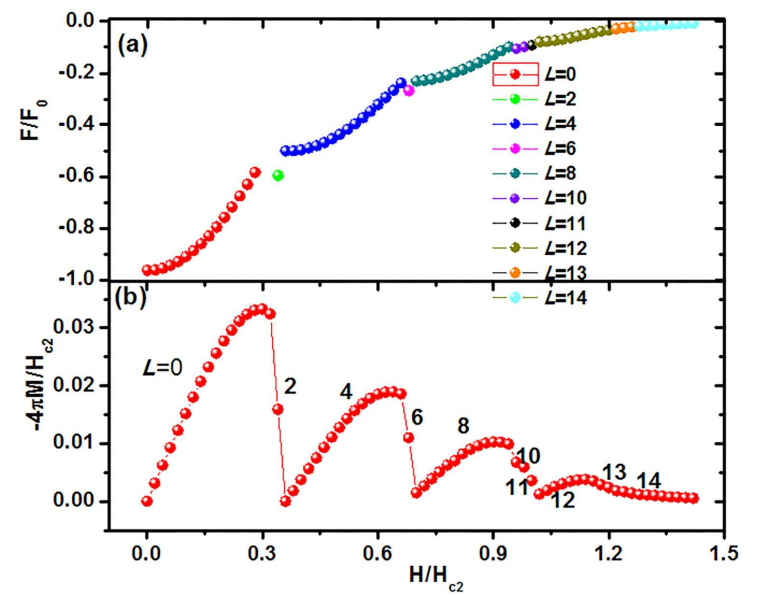

Fig. 6. Free-energy curve (a) and magnetization (b) for a mesoscopic superconducting cube of size $a=10 \xi$ with a single weak link of size $d=\xi$ in an external uniform magnetic field parallel to the $z$-axis.

For the further investigation on the structure of vortices, the magnetic field is kept perpendicular to the weak-link plane, and more specifically, parrallel to the $z$ axis $\left(H_{z} \equiv H\left(\theta=0^{\circ}\right)\right)$. Figure 6 shows the full freeenergy spectrum and magnetization as a function of applied magnetic field for the sample. Figure 6a shows the free energy as a function of $H$. The lowest energy curve corresponds to the thermodynamic stable pattern, the Meissner phase with no vortices, but only up to the first penetration field $H_{p}=0.32 H_{c 2}$. Above this field the entrances of two vortex tubes are favored (such as $\left.H=0.34 H_{c 2}\right)$. As the applied field is further raised, four vortex tubes enter the sample. Each jump in the magnetization curve corresponds to a transition between different vortex states (Fig. 6b). We find the vortex states with vorticity $L=2,4,6,8,10,11, \ldots, 14$. To investigate the effects of the magnetic field on the vortex states, the selected vortex states are given in Fig. 7. One can see that the vortices enter into the cube with increasing $H$ value. The strong confinement in the mesoscopic regime prevents the formation of hexagonal structures and we usually obtain ring symmetric structures. With increasing magnetic field vortex enters the sample in the form of a tube which was believed to be due to the presence of a surface barrier [19]. Furthermore, we were also able to observe the subtle changes of vortex tubes inside the weak link.

\section{Conclusions}

In this paper we solved the time-dependent GinzburgLandau equations for the three-dimensional mesoscopic superconductor with one central weak link. We obtained numerical expression in terms of the finite-element method. Our results show that the final distribution of vortices is determined by the competing interactions of 
vortices with the Meissner currents and the weak-link boundaries. With increase of magnetic field the vortex entered the sample in the form of tube which was believed to be due to the presence of a surface barrier. Furthermore, we were also able to observe the subtle changes of vortex tubes inside the weak link.

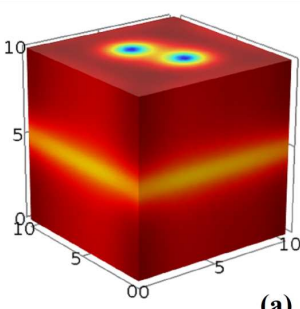

(a)

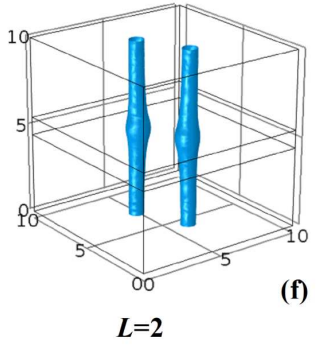

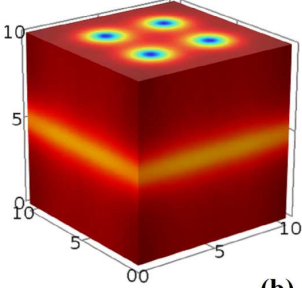

(b)

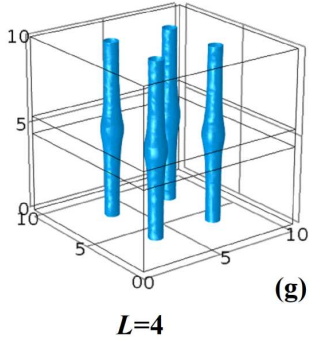

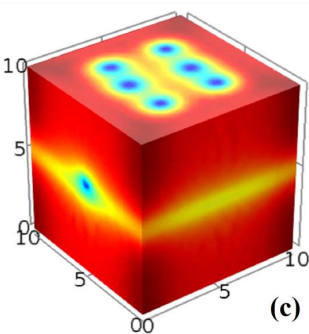

(c)

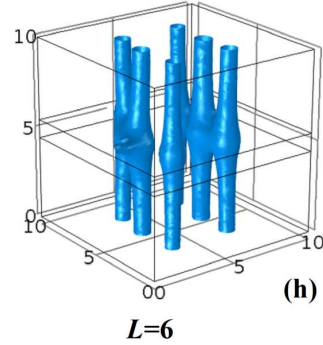

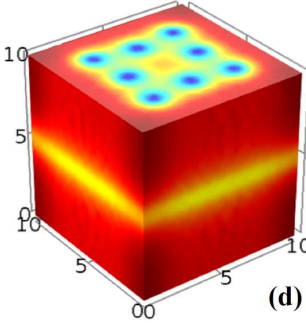
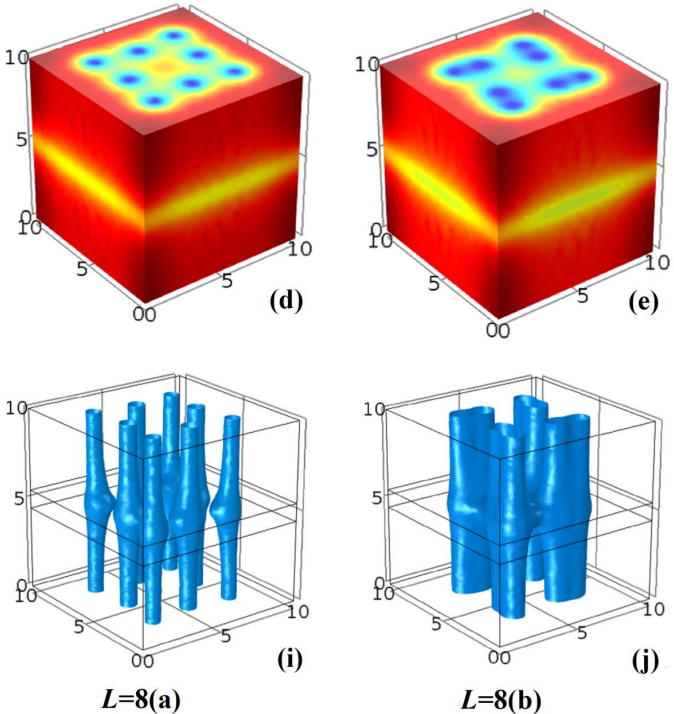

Fig. 7. Vortex tubes for a mesoscopic superconducting cube of size $a=10 \xi$ with a single weak link of size $d=\xi$ in an external uniform magnetic field parallel to the $z$-axis, at the field $\frac{H}{H_{c 2}}=0.34(\mathrm{a}), \frac{H}{H_{c 2}}=0.60$ (b), $\frac{H}{H_{c 2}}=0.68$ (c), $\frac{H}{H_{c 2}}=0.72(\mathrm{~d})$ and $\frac{H}{H_{c 2}}=0.80(\mathrm{e})$. Parts $(\mathrm{f})-(\mathrm{j})$ show the corresponding isosurface plots of vortex tubes of (a) $-(\mathrm{e})$. Blue to red means order parameter range from minimum to maximum.

\section{Acknowledgments}

This work is sponsored by National Natural Science Foundation of China (No. 51672172, 11502141, 51502168), the Opening Project of Shanghai Key Laboratory of High Temperature Superconductors (No. 14DZ2260700), the Natural Science Foundation of Shanghai (No. 17ZR1411400, 15ZR1418700).

\section{References}

[1] G. Carapella, P. Sabatino, G. Costabile, Phys. Rev. B 81, 054503 (2010).

[2] Ben Xu, M.V. Milošević, F.M. Peeters, Phys. Rev. B 81, 064501 (2010).

[3] T. Cren, L. Serrier-Garcia, F. Debontridder, D. Roditchev, Phys. Rev. Lett. 107, 097202 (2011).

[4] A. Kanda, B.J. Baelus, F.M. Peeters, K. Kadowaki, Y. Ootuka, Phys. Rev. Lett. 93, 257002 (2004).

[5] M.V. Milošević, A. Kanda, S. Hatsumi, F.M. Peeters, Y. Ootuka, Phys. Rev. Lett. 103, 217003 (2009).

[6] T. Winiecki, C.S. Adams, J. Comp. Phys. 179, 127 (2002).

[7] Ž. L. Jelić, M.V. Milošević, J. Van de Vondel, A.V. Silhanek, Sci. Rep. 5, 14604 (2015).
[8] L. Peng, Y. Hu, Z. Li, K. Deng, Y. Zhu, L. Xu, Y. Zhou, Acta Phys. Pol. A 133, 152 (2018).

[9] B.J. Baelus, F.M. Peeters, Phys. Rev. B 65, 104515 (2002).

[10] L. Peng, C. Cai, J. Low. Temp. Phys. 183, 371 (2016).

[11] L.F. Chibotaru, A. Ceulemans, V. Bruyndoncx, V.V. Moshchalkov, Nature (London) 408, 833 (2000).

[12] D.Y. Vodolazov, F.M. Peeters, S.V. Dubonos, A.K. Geim, Phys. Rev. B 67, 054506 (2003).

[13] I.V. Grigorieva, W. Escoffier, J. Richardson, L.Y. Vinnikov, S. Dubonos, V. Oboznov, Phys. Rev. Lett. 96, 077005 (2006).

[14] G. Carapella, P. Sabatino, C. Barone, S. Pagano, M. Gombos, Sci. Rep. 6, 35694 (2016).

[15] G. Deutscher, P.G. de Gennes, Superconductivity, Ed. R.D. Parks, Vol. 2, Marcel Dekker, New York 1969, Ch. 17.

[16] L. Peng, J. Lin, Y. Zhou, Y. Zhang, J. Supercond. Nov. Magn. 28, 3507 (2015).

[17] Q. Du, Phys. Rev. B 46, 9027 (1992).

[18] T.S. Alstrøm, M.P. Sørensen, N.F. Pedersen, S. Madsen, Acta. Appl. Math. 115, 63 (2011).

[19] A. Fortini, E. Paumier, Phys. Rev. B 14, 55 (1976). 